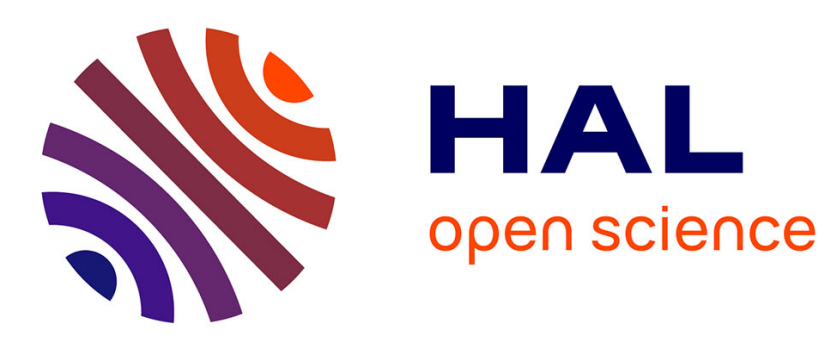

\title{
Application des méthodes de sélection récurrente à l'amélioration génétique de la Vigne
}

\author{
Alain Bouquet, Paul Truel, Robert Wagner
}

\section{To cite this version:}

Alain Bouquet, Paul Truel, Robert Wagner. Application des méthodes de sélection récurrente à l'amélioration génétique de la Vigne. Agronomie, 1981, 1 (1), pp.65-73. hal-00884224

\section{HAL Id: hal-00884224 \\ https://hal.science/hal-00884224}

Submitted on 1 Jan 1981

HAL is a multi-disciplinary open access archive for the deposit and dissemination of scientific research documents, whether they are published or not. The documents may come from teaching and research institutions in France or abroad, or from public or private research centers.
L'archive ouverte pluridisciplinaire HAL, est destinée au dépôt et à la diffusion de documents scientifiques de niveau recherche, publiés ou non, émanant des établissements d'enseignement et de recherche français ou étrangers, des laboratoires publics ou privés. 


\title{
Application des méthodes de sélection récurrente à l'amélioration génétique de la Vigne
}

\author{
Alain BOUQUET*, Paul TRUEL** et Robert WAGNER*** \\ * Station de Recherches de Viticulture, Domaine de la Grande-Ferrade, Centre de Recherches de Bordeaux, \\ I.N.R.A., 33140 Pont-de-la-Maye \\ ** Station de Recherches de Viticulture, Domaine de Vassal, Centre de Recherches de Montpellier, I.N.R.A., \\ 34340 Marseillan \\ *** Station de Recherches de Viticulture, Domaine du Chapitre, Centre de Recherches de Montpellier, \\ I.N.R.A., 34750 Villeneuve-lès-Maguelonne
}

RÉSUME

Vigne,

Sélection récurrente, Amélioration génétique. Couleur du vin.

\begin{abstract}
La nécessité de préserver la variabilité génétique au cours des programmes de sélection incite à concevoir une stratégie d'amélioration de la Vigne à long terme dans laquelle la sélection de nouvelles variétés est menée parallèlement à l'amélioration du matériel servant de base à cette sélection. Les modèles de sélection récurrente sont parfaitement adaptés à ce type de stratégie, à condition de tenir compte des contraintes imposées par la plante. La culture hydroponique en serre, qui permet une accélération considérable des générations, rend possible la réalisation rapide de plusieurs cycles de sélection récurrente pour certains caractères bien particuliers, notamment les caractères de résistance aux parasites. Cependant, pour de nombreux caractères, une sélection en serre n'est pas possible : il faut se résoudre à accepter les contraintes et à les intégrer dans un schéma de sélection d'une manière aussi rationnelle que possible et compatible avec la recherche d'une utilisation optimale des moyens. Dans cette optique, est proposé un programme de sélection récurrente dont le but est l'amélioration d'une population de Vitis vinifera qui servira de matériel de base à la création de nouvelles variétés bien adaptées aux conditions du vignoble méridional et aptes à produire des vins colorés de haute qualité.
\end{abstract}

\begin{abstract}
Grapevine,

Recurrent selection,

Breeding,

Wine colour.

\section{Recurrent selection in Grapevine breeding}

The need to keep enough genetic variability preserved leads to imagine long term grape breeding programs in which the selection of new cultivars would be carried out in parallel with the improvement of the germplasm which is the base of this selection. Accordingly a recurrent selection program started off in 1977 at Montpellier. Its aim is the improvement of a Vitis vinifera set of genotypes which will be used, after each selection process, as germplasm for breeding new varieties, able to give good quality and colored wines, and which would be well adapted to French Southern vineyards. Other applications of recurrent selection in grape breeding are discussed, in relation with the use of hydroponic culture under greenhouse conditions.
\end{abstract}

\section{INTRODUCTION}

Jusqu'à présent, la stratégie des sélectionneurs de Vigne ayant recours aux méthodes d'amélioration par voie sexuée consistait à obtenir, à partir des cépages existants, une variabilité génétique nouvelle qui était exclusivement exploitée pour la création variétale, la multiplication végétative permettant de fixer et de diffuser sous forme monoclonale les structures génétiques les plus performantes apparues au cours des croisements.

Cette attitude, qui répond à un souci d'efficacité, a permis des progrès certains dans l'amélioration de la gamme variétale proposée aux viticulteurs. Ces progrès se sont récemment traduits en France par l'inscription au catalogue de plusieurs variétés nouvelles de cuve et de table ainsi que d'une variété porte-greffes (variétés I.N.R.A.), qui devraient contribuer à la solution de certains problèmes d'adaptation à des conditions climatiques, édaphiques et culturales bien particulières, tout en améliorant sensiblement la qualité des produits obtenus (DURQUETY, 1978 ; Pouget et OtTenwaelter, 1978 ; Truel et Vergnes, 1978).

On peut cependant s'interroger sur l'efficacité à long terme d'une telle méthode. En effet, la variabilité génétique initiale, représentée pratiquement par les collections ampélographiques, n'est pas inépuisable, d'autant plus qu'une partie importante de celle-ci reste inexploitée : de nombreuses variétés qui pourraient être une réserve de gènes intéressants ne sont pas utilisées comme géniteurs, ou sont rapidement abandonnées pour la simple raison qu'elles ne donnent pas en croisement des descendances ayant une valeur culturale suffisante.

Lorsque, pour un objectif de sélection donné, la variabilité de départ est complètement exploitée, l'attitude logique 
consiste à repartir des variétés améliorées ainsi obtenues, à les croiser entre elles de manière à créer une nouvelle variabilité qui est soumise à un deuxième cycle de sélection. Dans certains cas, le recours à des cycles successifs de sélection est rendu indispensable par le niveau insuffisant de telle ou telle caractéristique dans les premières variétés améliorées obtenues. On peut citer comme exemple la qualité organoleptique des hybrides producteurs directs créés en France dans la première moitié du $\mathrm{XX}^{\mathrm{e}}$ siècle.

Le problème qui se pose alors est que la perte de variabilité entraînée par la création variétale apparaît incompatible avec la nécessité d'entretenir cette variabilité à un niveau suffisant afin de préserver les chances de progrès génétique au cours des cycles futurs de sélection.

\section{PRINCIPES D'UNE STRATÉgIE GÉNÉRALE À LONG TERME}

DE L'AMÉLIORATION GENÉTIQUE DE LA VIGNE

Il est possible de définir une stratégie générale à long terme de l'amélioration génétique de la Vigne, dans laquelle la sélection des nouvelles variétés est menée parallèlement à l'amélioration du matériel servant de base à cette sélection, conformément au modèle général proposé par GALLAIS (1977).

\section{a) Amélioration du matériel de base}

L'amélioration du matériel de base, menée de façon récurrente, vise à augmenter le niveau moyen de la population, pour le ou les caractères pris en considération, tout en préservant la variabilité génétique de départ. La succession de plusieurs cycles d'amélioration favorise en outre la coadaptation des gènes et permet ainsi une meilleure utilisation des effets d'épistasie. Or Rives (1977) a souligné l'importance de ces effets chez une plante à multiplication végétative comme la Vigne. Cette coadaptation des gènes revêt une grande importance dans le cas d'introgression de caractères de résistance oligo- ou polyfactoriels à partir de matériel d'origine interspécifique, ou dans l'utilisation en sélection de mutations géniques ou chromosomiques obtenues artificiellement.

Le système de reproduction doit être conçu de manière à maximiser les probabilités d'obtention d'associations géniques favorables, tout en contrôlant les effets d'inbreeding rendus inévitables par le nombre forcément limité des variétés constituant la population de départ. La Vigne, plante fortement hétérozygote, est en effet très sensible à la consanguinité qui se traduit le plus souvent par une baisse considérable de vigueur et de fertilité.

Les opérateurs de sélection basés sur des critères génotypiques et notamment sur des tests de descendances doivent être privilégiés par rapport aux simples critères phénotypiques. En effet, l'amélioration du matériel de base porte plus sur l'aptitude à transmettre un caractère intéressant que sur ce caractère lui-même, puisque les génotypes extraits de la population de base serviront essentiellement de géniteurs dans les cycles de création variétale.

\section{b) Création variétale}

Les opérateurs de sortie vers la création variétale peuvent être relativement simples : la multiplication végétative suivie de sélection clonale permet en effet de fixer et de diffuser les combinaisons géniques intéressantes apparues au cours des cycles d'amélioration du matériel de base. En fait, le matériel de base étant constitué de géniteurs potentiels, les opérateurs de sortie devront, la plupart du temps être plus élaborés et faire intervenir au moins une génération sexuée:

- croisements entre génotypes extraits de la population de base et variétés cultivées connues pour leur aptitude à donner des descendances performantes du point de vue cultural et qualitatif,

- croisements entre génotypes issus d'autofécondations pratiquées dans la population de base. On bénéficie ainsi (DEMARLY, 1972) de l'augmentation de variance des aptitudes à la combinaison due à la consanguinité et, éventuellement, de meilleures probabilités de recombinaison génétique (PANDEY, 1972)

- combinaison des deux opérateurs précédents, c'est-àdire croisements entre des variétés cultivées et des génotypes issus d'autofécondations pratiquées dans la population de base.

\section{c) Limites d'une telle stratégie}

Elles sont nombreuses et liées essentiellement aux contraintes générales posées par la sélection des espèces ligneuses et pérennes. Parmi celles-ci, on peut citer la longueur des cycles de générations, la première mise à fruits se produisant quatre à cinq ans après le semis, en conditions de culture normales; l'importance des surfaces nécessaires à l'entretien et à l'étude des populations, la densité courante de plantation étant de 3000 à 5000 pieds à l'hectare; la difficulté, voire l'impossibilité d'établir des corrélations jeune-adulte pour de nombreux caractères économiquement importants, notamment ceux relatifs à l'équilibre rendement-qualité.

Face à cette situation, on peut proposer deux types de programmes d'amélioration génétique basés sur la sélection récurrente :

- Un programme accéléré, où les cycles d'amélioration du matériel de base sont menés intégralement en serre (BOUQUET, 1977), dans la mesure naturellement où les caractères soumis à sélection s'y prêtent.

- Un programme plus classique, où les cycles d'amélioration du matériel de base et les cycles de création variétale sont menés au vignoble. En effet, si l'on envisage un programme d'amélioration pour des caractères ayant trait à l'équilibre rendement-qualité, la sélection en serre n'est pas possible: il faut se résoudre à accepter les contraintes imposées par la plante et à les intégrer dans un schéma de sélection récurrente d'une manière aussi rationnelle que possible et compatible avec la recherche d'une utilisation optimale des moyens.

\section{SÉLECTION RÉCURRENTE ACCÉLÉRÉE EN SERRE}

Les avantages d'un tel modèle sont essentiellement liés à l'accélération des générations rendue possible par la culture hydroponique en serre. La première mise à fruits ayant lieu 16 à 18 mois après le semis, la Vigne peut à la limite être traitée comme un plante bisannuelle, ce qui permet un raccourcissement considérable des cycles d'amélioration du matériel de base. Les résultats récents de SRINIVASAN et MULLINS (1979) concernant la conversion de vrilles en inflorescences au moyen de régulateurs de croissance (cytokinines) ouvrent des perspectives encore plus intéressantes dans ce domaine, en permettant théoriquement d'obtenir des mises à fruits en moins de 6 mois. 


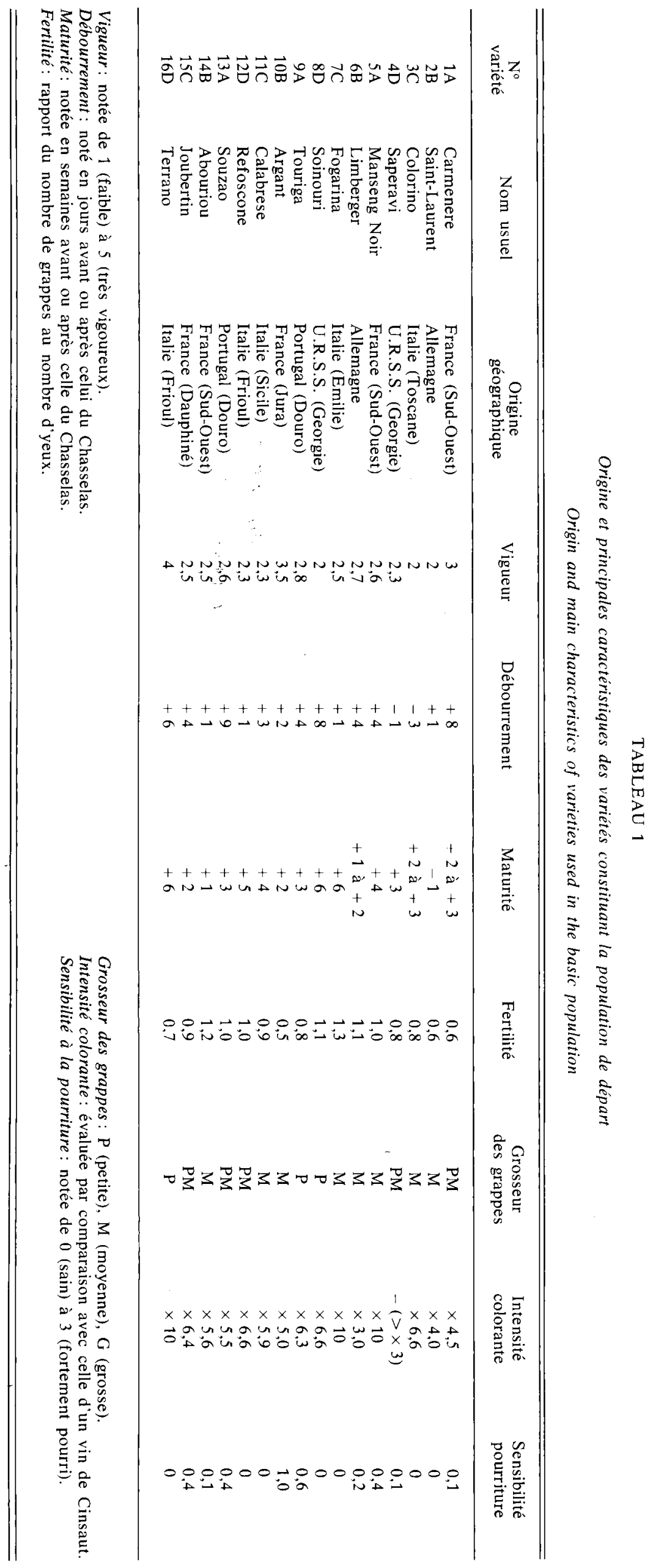


Cette accélération des générations rend possible l'intégration d'une génération d'autofécondation dans chaque cycle de sélection récurrente, d'autant plus que la dépression de vigueur observée sur des lignées autofécondées de Vigne est moins marquée en serre qu'au vignoble. L'intérêt de ces autofécondations a été discuté dans un précédent article (BOUQUET, 1977).

La principale restriction est que l'amélioration du matériel de base ne peut être évidemment envisagée que pour des caractères mesurables en serre et dont les corrélations jeune-adulte et serre-vignoble sont suffisamment élevées: fertilité (WAGNER et BRONNER, 1974), dimension des grappes et des baies, peut-être efficacité photosynthétique (CARBONNEAU et al., 1980), mais surtout résistance aux parasites, quelles que soient d'ailleurs les origines intra- ou interspécifiques des caractères de résistance pris en considération.

On peut, en particulier, envisager l'utilisation de cette méthode de sélection récurrente en serre pour améliorer une population "tampon", constituée de géniteurs d'origine interspécifique ou intergénérique et notamment issus d'hybridation Vitis $\times$ Muscadinia (BovQUeT, 1980), de manière à permettre une intégration optimale des gènes de résistance dans une population de Vitis vinifera dont l'amélioration serait menée parallèlement au vignoble.

On peut également envisager (HEwITT et RIVES, 1979) d'utiliser cette méthode pour élever le niveau de résistance horizontale d'une population de Vitis vinifera vis-à-vis d'un parasite cryptogamique comme le Mildiou (Plasmopara viticola). En effet, les variétés de Vitis vinifera, qui sont toutes également sensibles au parasite en conditions d'infection "sévères", se différencient notablement par leur comportement en conditions d'infection "douces" (crible phénotypique), et encore plus nettement lorsqu'elles sont croisées avec un testeur résistant d'origine interspécifique et que leurs descendances sont testées en conditions « sévères " (crible génotypique) : la fréquence des plantes résistantes dans les descendances est en effet très variable et dépend du génotype Vitis vinifera utilisé (DOAZAN et KıM, 1978).

Si l'on admet que les allèles favorables mis en évidence par les deux cribles sont identiques, un programme de sélection récurrente constitué par une succession de top-crosses, tests de résistance en conditions "sévères" (plus faciles à obtenir que les conditions «douces»), et intercroisement des plantes sélectionnées en fonction de la résistance moyenne de leurs descendances, devrait permettre d'augmenter la fréquence moyenne de ces allèles favorables et donc la résistance horizontale de la population de Vitis vinifera jusqu'à un niveau tel que des différences phénotypiques apparaissent en conditions d'infection "sévères". Il va de soi qu'un tel programme implique la réalisation d'un nombre relativement élevé de cycles de sélection et ne peut donc se concevoir qu'en ayant recours aux techniques d'accélération des générations.

\section{SÉLECTION RECURRENTE AU VIGNOBLE : UN EXEMPLE CONCRET}

\section{a) Objectif de sélection}

L'objectif du programme de sélection récurrente, entamé en 1977 à la Station de recherches viticoles de Montpellier, est l'amélioration d'une population de Vitis vinifera devant servir de matériel de base à la création de nouvelles variétés aptes à produire des vins colorés de haute qualité et bien adaptées aux conditions du vignoble méridional. En effet, l'obtention d'une couleur soutenue est indispensable à la commercialisation des vins de table du Midi et les insuffisances de l'encépagement actuel dans ce domaine ne sont que partiellement compensées par l'utilisation de cépages «teinturiers", c'est-à-dire à jus coloré, dont le niveau qualitatif est assez médiocre.

\section{b) Choix du matériel de départ}

Le choix des variétés constituant la population de départ est primordial et répond à deux exigences :

- La variabilité génétique de l'espèce Vitis vinifera doit être convenablement représentée par l'incorporation de cépages d'origines géographiques aussi diverses que possible.

- Le nombre de variétés constituant la population de départ doit être aussi élevé que le permettent les contraintes matérielles.

La population de départ $\left(\mathrm{P}_{0}^{\prime}\right)$ est donc constituée de 16 variétés de Vitis vinifera, non teinturières, choisies parmi les 781 variétés disponibles dans les collections ampélographiques du domaine de Vassal, collections qu'on peut assimiler à une population $\mathrm{P}_{0}$. La sélection, purement phénotypique, a porté exclusivement sur l'aptitude à donner des vins colorés. Les autres caractéristiques, phénologiques, culturales et qualitatives sont très variables (tabl. 1 et annexe). Ces 16 variétés ont été réparties en 4 groupes géographiques, $\mathrm{A}$ (régions océaniques), $\mathrm{B}$ (régions septentrionales et subcontinentales). C (Italie et Savoie) et D (Adriatique et mer Noire).

\section{c) Système de reproduction}

L'utilisation d'un plan diallèle complet n'étant pas envisageable du point de vue pratique, les 16 variétés ont été intercroisées suivant un schéma diallèle partiel (GORDON, 1980) : chaque variété a été croisée avec six autres variétés, soit comme mâle (ex.: Limberger, Saint-Laurent), soit comme femelle (ex. : Manseng Noir, Souzao), soit à la fois comme mâle et femelle. Pour chaque combinaison du diallèle partiel, le sens du croisement a en effet été imposé par les contraintes de phénologie florale. On a utilisé la méthode des croisements par permutation circulaire en prenant soin de ne croiser que des variétés appartenant à des groupes différents, de façon à minimiser les risques d'inbreeding (fig. 1).

La réalisation de ce diallèle partiel qui s'est étalée sur deux ans (1978 et 1979) a donc produit 48 familles de pleins frères qui constituent la population $\mathrm{P}_{1}$ (tabl. 2). Cette population, qui possède une fréquence de gènes favorables à la couleur identique à celle de la population $\mathrm{P}_{0}^{\prime}$, est soumise à une sélection combinée inter et intrafamilles.

\section{d) Opérateurs de sélection}

La sélection interfamilles est en fait une sélection génotypique qui porte sur l'aptitude à donner des vins colorés avec un rendement économiquement acceptable. On ne conserve que les familles dont l'un au moins des parents a été retenu en fonction de la valeur de ses descendances en croisement avec les variétés Grenache et Cabernet-Sauvignon utilisées comme testeurs (fig. 2). La réalisation du top-cross en 1977 a donc produit 32 familles de pleins frères (tabl. 3). La valeur moyenne de ces familles est estimée au moyen d'un 


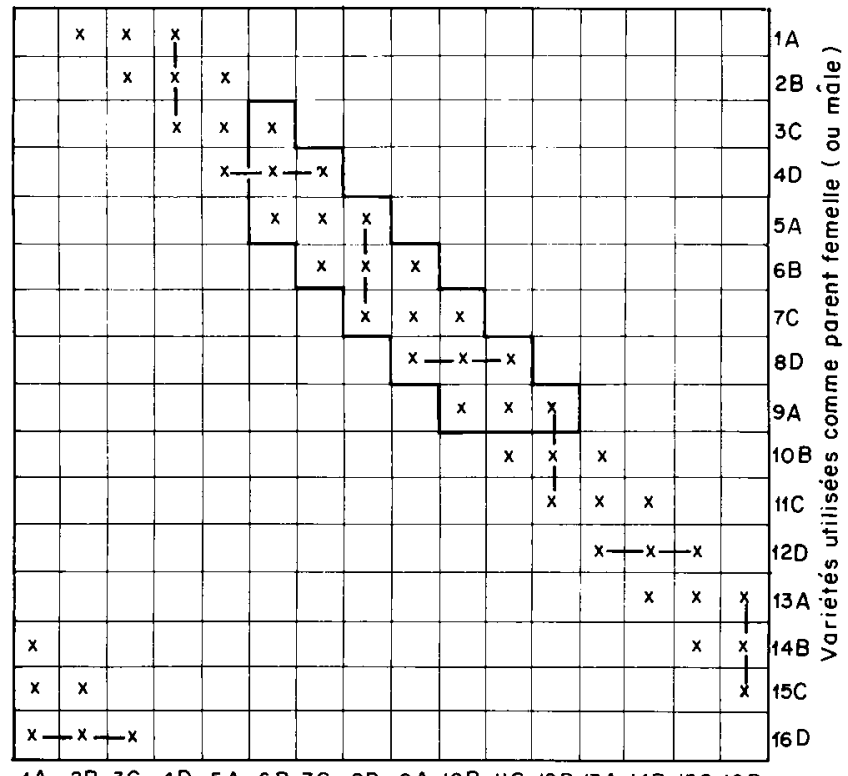

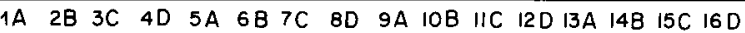
Variétés utilisées comme parentmôle (ou femelle)

Figure 1

Réalisation du diallèle partiel par permutation circulaire.

Minimum de 18 familles conservées en fonction des résultats du top-cross: cas où les 4 variétés retenues (ex.: $6 \mathrm{~B}, 7 \mathrm{C}, 8 \mathrm{D}, 9 \mathrm{~A}$ ) appartiennent à quatre groupes géographiques différents. Familles encadrées d'un trait gras.

Maximum de 24 familles conservées: cas où les quatre variétés retenues (ex.: $4 D, 8 D, 12 D, 16 D$ ) appartiennent au même groupe géographique. Familles reliées d'un trait gras.

index de sélection faisant intervenir le taux d'anthocyanes facilement extractibles dans les pellicules, le taux de sucres dans les moûts, le poids de récolte par souche.

Si le nombre de variétés retenues en fonction des résultats du top-cross est $\mathrm{N}=4$, on aura un minimum de 18 et un maximum de 24 familles conservées sur les 48 de la population $\mathrm{P}_{1}$, soit une pression de sélection relativement faible qui correspond au souci de maintenir une variabilité génétique élevée. Le minimum de 18 familles conservées correspond au cas où les 4 variétés retenues appartiendraient aux 4 groupes géographiques. Le maximum de 24 familles conservées correspond au cas où les 4 variétés retenues appartiendraient au même groupe géographique (fig. 1).

La sélection intrafamilles est une sélection phénotypique qui porte sur l'aptitude à donner des vins colorés, mais également sur l'ensemble des caractères pris en compte dans les opérations de sélection de la Vigne (fertilité, rusticité, maturité précoce ou moyenne, qualité, etc...). La pression de sélection est donc élevée, d'autant plus que le nombre de plantes retenues par famille est limité par les possibilités pratiques de réalisation du top-cross au cours du $2^{\mathrm{e}}$ cycle de sélection récurrente. En fait, ce nombre ne pourra pas être supérieur à l'unité.

On obtiendra ainsi à la fin du $1^{\text {er }}$ cycle de sélection, une population $\mathrm{P}_{1}^{\prime}$, constituée de 18 à 24 génotypes. Ces génotypes, beaucoup mieux adaptés aux conditions du vignoble méridional que ne l'étaient les variétés de la population $\mathrm{P}_{0}$, seront à leur tour soumis à un top-cross, puis intercroisés toujours selon la méthode du diallèle partiel, mais avec un contrôle des appariements, de façon à limiter la consanguinité et à maximiser les recombinaisons génétiques : Dans la mesure du possible, on ne croisera que des plantes issues de parents différents. De plus, chaque génotype sera croisé avec seulement 4 génotypes différents, au lieu de 6 pour le premier diallèle partiel. La population $\mathrm{P}_{2}$ ainsi obtenue, constituée de 36 à 48 familles est donc de taille équivalente à celle de la population $P_{1}$. Comme cette dernière, elle sera soumise à une sélection inter et intrafamilles, et ainsi de suite..

\section{e) Discussion}

Le fait de réaliser le diallèle partiel sans attendre les résultats du top-cross réduit pratiquement de moitié la durée de chaque cycle de sélection récurrente. En outre, ce système de reproduction préserve assez bien la variabilité génétique en passant d'un cycle à un autre et peut éventuellement fournir une information génétique complémentaire de celle du top-cross (PEDERSON, 1980), bien que l'interprétation statistique d'un dispositif diallèle partiel soit assez complexe.

En contrepartie, la réalisation quasi-simultanée du top-cross et du diallèle partiel demande un travail de croisements supplémentaire et des surfaces immobilisées plus grandes. De plus, le progrès génétique par cycle n'est pas maximisé, puisque les populations $\mathrm{P}^{\prime}$ successives sont composées en majorité de génotypes dont un seul des parents a été sélectionné sur la valeur de ses descendances. Cependant, le progrès génétique par année devrait être comparable à celui attendu dans un programme de sélection récurrente classique où le choix des géniteurs entrant dans le système de reproduction est déterminé par l'analyse de leurs descendances en croisement.

Le choix d'une méthode de sélection basée sur la valeur de descendances obtenues en croisement avec des testeurs, est aussi motivé par le souci de combiner de manière optimale les opérations de création variétale et les opérations d'amélioration du matériel de base, également consommatrices en temps de travail et superficies (fig. 2). En effet, les top-crosses, qui constituent les opérateurstests des cycles de sélection récurrente, sont des opérateurs de sortie privilégiés pour la création de nouvelles variétés. C'est la raison pour laquelle les variétés Grenache et Cabernet-Sauvignon, connues pour la qualité de leur vin et leur aptitude à donner des descendances performantes, ont été choisies comme testeurs.

Le schéma proposé permet d'envisager une sélection récurrente réciproque, en partant de deux populations distinctes, l'une sélectionnée pour l'aptitude à donner des vins colorés, l'autre sélectionnée par exemple pour la résistance à la pourriture grise (Botrytis cinerea). Au fur et à mesure du développement de la sélection, les testeurs peuvent évoluer, les nouvelles variétés sélectionnées dans l'un des programmes devenant les testeurs utilisés dans l'autre programme.

\section{CONCLUSION}

En conclusion, il convient de mettre l'accent sur le caractère perfectible de ces conceptions qui peuvent apparaître pour l'instant assez théoriques. En fait, la méthodologie de la sélection la plus rationnelle se précisera au fur et à mesure de la réalisation de programmes de ce type, en fonction des contraintes et des difficultés qui ne manqueront pas d'apparaître, et grâce à l'information génétique rendue disponible, qui permettra en particulier de moduler les pressions de sélection effectivement appliquées. 
TABLEAU 2

Résultats du diallèle partiel (Années 1978 et 1979)

Results of the partial diallel

\begin{tabular}{|c|c|c|c|c|c|c|}
\hline Parent femelle & Parent mâle & $\begin{array}{l}\mathrm{N}^{0} \mathrm{du} \\
\text { croisement }\end{array}$ & $\begin{array}{c}\mathrm{Nb} \text { de } \\
\text { grappes } \\
\text { pollinisées }\end{array}$ & $\begin{array}{l}\text { Nb de } \\
\text { pépins } \\
\text { obtenus }\end{array}$ & $\begin{array}{c}\text { p. } 100 \\
\text { germination }\end{array}$ & $\begin{array}{c}\text { Nb de } \\
\text { plantes } \\
\text { disponibles }\end{array}$ \\
\hline Carmenere & $\begin{array}{l}\times \text { Saint-Laurent } \\
\times \text { Colorino } \\
\times \text { Abouriou } \\
\times \text { Joubertin } \\
\times \text { Terrano }\end{array}$ & $\begin{array}{l}(1 \mathrm{~A} \times 2 \mathrm{~B}) \\
(1 \mathrm{~A} \times 3 \mathrm{C}) \\
(1 \mathrm{~A} \times 14 \mathrm{~B}) \\
(1 \mathrm{~A} \times 15 \mathrm{C}) \\
(1 \mathrm{~A} \times 16 \mathrm{D})\end{array}$ & $\begin{array}{l}5 \\
6 \\
8 \\
6 \\
7\end{array}$ & $\begin{array}{l}708 \\
540 \\
615 \\
535 \\
565\end{array}$ & $\begin{array}{l}43 \\
52 \\
46 \\
53 \\
53\end{array}$ & $\begin{array}{l}240 \\
240 \\
240 \\
250 \\
270\end{array}$ \\
\hline Colorino & $\begin{array}{l}\times \text { Saint-Laurent } \\
\times \text { Limberger } \\
\times \text { Terrano }\end{array}$ & $\begin{array}{l}(3 \mathrm{C} \times 2 \mathrm{~B}) \\
(3 \mathrm{C} \times 6 \mathrm{~B}) \\
(3 \mathrm{C} \times 16 \mathrm{D})\end{array}$ & $\begin{array}{l}3 \\
3 \\
2\end{array}$ & $\begin{array}{l}810 \\
608 \\
810\end{array}$ & $\begin{array}{l}32 \\
42 \\
64\end{array}$ & $\begin{array}{l}210 \\
226 \\
400\end{array}$ \\
\hline Saperavi & $\begin{array}{l}\times \text { Carmenere } \\
\times \text { Saint-Laurent } \\
\times \text { Colorino } \\
\times \text { Limberger }\end{array}$ & $\begin{array}{l}(4 D \times 1 A) \\
(4 D \times 2 B) \\
(4 D \times 3 C) \\
(4 D \times 6 B)\end{array}$ & $\begin{array}{l}5 \\
5 \\
7 \\
6\end{array}$ & $\begin{array}{l}512 \\
570 \\
354 \\
486\end{array}$ & $\begin{array}{l}53 \\
64 \\
70 \\
70\end{array}$ & $\begin{array}{l}220 \\
280 \\
190 \\
300\end{array}$ \\
\hline Manseng noir & $\begin{array}{l}\times \text { Saint-Laurent } \\
\times \text { Colorino } \\
\times \text { Saperavi } \\
\times \text { Limberger } \\
\times \text { Fogarina } \\
\times \text { Soinouri }\end{array}$ & $\begin{array}{l}(5 \mathrm{~A} \times 2 \mathrm{~B}) \\
(5 \mathrm{C} \times 3 \mathrm{C}) \\
(5 \mathrm{~A} \times 4 \mathrm{D}) \\
(5 \mathrm{~A} \times 6 \mathrm{~B}) \\
(5 \mathrm{~A} \times 7 \mathrm{C}) \\
(5 \mathrm{~A} \times 8 \mathrm{D})\end{array}$ & $\begin{array}{r}6 \\
3 \\
2 \\
8 \\
4 \\
12\end{array}$ & $\begin{array}{l}360 \\
608 \\
608 \\
540 \\
426 \\
634\end{array}$ & $\begin{array}{l}80 \\
70 \\
75 \\
68 \\
68 \\
72\end{array}$ & $\begin{array}{l}230 \\
392 \\
385 \\
285 \\
224 \\
370\end{array}$ \\
\hline Fogarina & $\begin{array}{l}\times \text { Saperavi } \\
\times \text { Limberger } \\
\times \text { Soinouri }\end{array}$ & $\begin{array}{l}(7 \mathrm{C} \times 4 \mathrm{D}) \\
(7 \mathrm{C} \times 6 \mathrm{~B}) \\
(7 \mathrm{C} \times 8 \mathrm{D})\end{array}$ & $\begin{array}{l}4 \\
6 \\
4\end{array}$ & $\begin{array}{l}323 \\
431 \\
364\end{array}$ & $\begin{array}{l}58 \\
82 \\
75\end{array}$ & $\begin{array}{l}152 \\
300 \\
253\end{array}$ \\
\hline Soinouri & $\begin{array}{l}\times \text { Limberger } \\
\times \text { Touriga } \\
\times \text { Argant } \\
\times \text { Calabrese }\end{array}$ & $\begin{array}{l}(8 \mathrm{D} \times 6 \mathrm{~B}) \\
(8 \mathrm{D} \times 9 \mathrm{~A}) \\
(8 \mathrm{D} \times 10 \mathrm{~B}) \\
(8 \mathrm{D} \times 11 \mathrm{C})\end{array}$ & $\begin{array}{l}8 \\
8 \\
7 \\
6\end{array}$ & $\begin{array}{l}684 \\
353 \\
500 \\
593\end{array}$ & $\begin{array}{l}92 \\
67 \\
62 \\
68\end{array}$ & $\begin{array}{l}340 \\
185 \\
270 \\
320\end{array}$ \\
\hline Touriga & $\begin{array}{l}\times \text { Limberger } \\
\times \text { Fogarina } \\
\times \text { Refoscone }\end{array}$ & $\begin{array}{l}(9 \mathrm{~A} \times 6 \mathrm{~B}) \\
(9 \mathrm{~A} \times 7 \mathrm{C}) \\
(9 \mathrm{~A} \times 12 \mathrm{D})\end{array}$ & $\begin{array}{l}9 \\
7 \\
5\end{array}$ & $\begin{array}{l}461 \\
457 \\
499\end{array}$ & $\begin{array}{l}82 \\
74 \\
80\end{array}$ & $\begin{array}{l}320 \\
290 \\
400\end{array}$ \\
\hline Argant & $\begin{array}{l}\times \text { Fogarina } \\
\times \text { Touriga } \\
\times \text { Calabrese } \\
\times \text { Refoscone }\end{array}$ & $\begin{array}{l}(10 \mathrm{~B} \times 7 \mathrm{C}) \\
(10 \mathrm{~B} \times 9 \mathrm{~A}) \\
(10 \mathrm{~B} \times 11 \mathrm{C}) \\
(10 \mathrm{~B} \times 12 \mathrm{D})\end{array}$ & $\begin{array}{l}7 \\
7 \\
4 \\
7\end{array}$ & $\begin{array}{l}507 \\
564 \\
420 \\
450\end{array}$ & $\begin{array}{l}50 \\
44 \\
65 \\
57\end{array}$ & $\begin{array}{l}210 \\
220 \\
249 \\
210\end{array}$ \\
\hline Calabrese & $\begin{array}{l}\times \text { Touriga } \\
\times \text { Refoscone } \\
\times \text { Abouriou }\end{array}$ & $\begin{array}{l}(11 \mathrm{C} \times 9 \mathrm{~A}) \\
(11 \mathrm{C} \times 12 \mathrm{D}) \\
(11 \mathrm{C} \times 14 \mathrm{~B})\end{array}$ & $\begin{array}{l}5 \\
7 \\
6\end{array}$ & $\begin{array}{l}383 \\
319 \\
389\end{array}$ & $\begin{array}{l}81 \\
77 \\
85\end{array}$ & $\begin{array}{l}218 \\
210 \\
290\end{array}$ \\
\hline Refoscone & $\times$ Joubertin & $(12 \mathrm{D} \times 15 \mathrm{C})$ & 12 & 324 & 76 & 250 \\
\hline Souzao & $\begin{array}{l}\times \text { Argant } \\
\times \text { Calabrese } \\
\times \text { Refoscone } \\
\times \text { Abouriou } \\
\times \text { Joubertin } \\
\times \text { Terrano }\end{array}$ & $\begin{array}{l}(13 \mathrm{~A} \times 10 \mathrm{~B}) \\
(13 \mathrm{~A} \times 11 \mathrm{C}) \\
(13 \mathrm{~A} \times 12 \mathrm{D}) \\
(13 \mathrm{~A} \times 14 \mathrm{~B}) \\
(13 \mathrm{~A} \times 15 \mathrm{C}) \\
(13 \mathrm{~A} \times 16 \mathrm{D})\end{array}$ & $\begin{array}{l}8 \\
5 \\
5 \\
6 \\
6 \\
5\end{array}$ & $\begin{array}{l}946 \\
259 \\
810 \\
414 \\
510 \\
850\end{array}$ & $\begin{array}{l}86 \\
84 \\
83 \\
86 \\
91 \\
86\end{array}$ & $\begin{array}{l}670 \\
141 \\
540 \\
244 \\
405 \\
630\end{array}$ \\
\hline Abouriou & $\begin{array}{l}\times \text { Refoscone } \\
\times \text { Joubertin }\end{array}$ & $\begin{array}{l}(14 \mathrm{~B} \times 12 \mathrm{D}) \\
(14 \mathrm{~B} \times 15 \mathrm{C})\end{array}$ & $\begin{array}{l}5 \\
4\end{array}$ & $\begin{array}{l}810 \\
810\end{array}$ & $\begin{array}{l}69 \\
61\end{array}$ & $\begin{array}{l}450 \\
400\end{array}$ \\
\hline Joubertin & $\times$ Saint-Laurent & $(15 \mathrm{C} \times 2 \mathrm{~B})$ & 6 & 820 & 18 & 120 \\
\hline Terrano & $\begin{array}{l}\times \text { Saint-Laurent } \\
\times \text { Abouriou } \\
\times \text { Joubertin }\end{array}$ & $\begin{array}{l}(16 \mathrm{D} \times 2 \mathrm{~B}) \\
(16 \mathrm{D} \times 14 \mathrm{~B}) \\
(16 \mathrm{D} \times 15 \mathrm{C})\end{array}$ & $\begin{array}{r}6 \\
14 \\
7\end{array}$ & $\begin{array}{l}810 \\
549 \\
699\end{array}$ & $\begin{array}{l}66 \\
55 \\
52\end{array}$ & $\begin{array}{l}430 \\
270 \\
340\end{array}$ \\
\hline
\end{tabular}

\section{ANNEXE}

Principales caractéristiques et aires de culture des variétés constituant la population de départ (ordre alphabétique)

\section{Abouriou}

Variété assez vigoureuse, de bonne fertilité, à grappes assez grosses, peu sensibles à la pourriture par suite d'une maturité précoce. Débourrement précoce. Cultivée seulement dans le Sud-Ouest et rencontrée dans l'Yonne. Donne un vin riche en tannins.

\section{Argant}

Variété très vigoureuse, de fertilité moyenne, à grappes assez grandes assez sensibles à la pourriture. Débourrement précoce. Cépage secondaire du Jura (Salins) qui n'est pratiquement plus cultivé par suite d'une maturité souvent trop tardive.

\section{Calabrese}

Variété assez vigoureuse, de fertilité moyenne, à grandes grappes peu sensibles à la pourriture. Débourrement moyen 

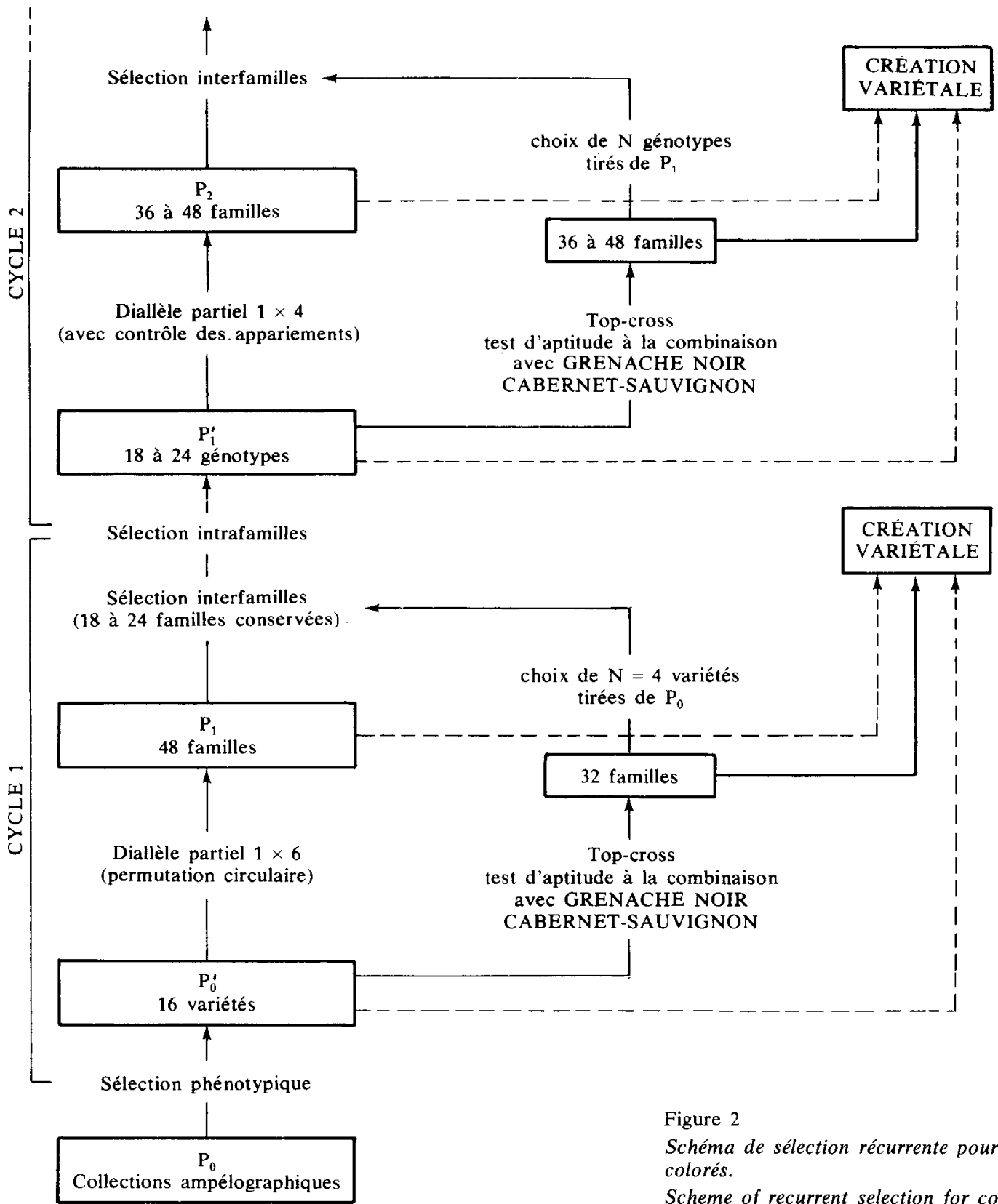

Figure 2

Schéma de sélection récurrente pour l'aptitude à donner des vins colorés.

Scheme of recurrent selection for coloured wines.

comme le Grenache. Cultivée en Sicile, surtout dans les provinces de Syracuse, de Ragusa, Catania, Enna et Palerme. En général vinifiée seule, donne des vins de coupage très alcooliques, tels que Pachino, Vittoria ou Scoglitti.

\section{Carmenere}

Variété du groupe des Cabernets, vigoureuse mais peu fertile, à grappes lâches et peu sensibles à la pourriture. Autrefois, un peu cultivée en Médoc pour la finesse de son vin.

\section{Colorino}

Variété de vigueur et fertilité moyennes, grappes à petites baies peu sensibles à la pourriture. Débourrement très précoce. Cultivée en Toscane, dans les provinces de Arezzo, Firenze, Pistoia, Siena, comme cépage apportant la couleur. N'est pas vinifiée seule.

\section{Fogarina}

Variété de vigueur moyenne et de bonne fertilité, à grappes assez lâches et peu sensibles à la pourriture. Débourrement assez précoce. Cultivée dans la province de Reggio Emilia, donne des vins de coupage.

\section{Joubertin}

Variété de vigueur et de fertilité moyennes, peu sensible à la pourriture. Débourrement assez précoce, comme l'Aramon. Un peu cultivée dans le département de l'Isère, donne un vin de faible degré alcoolique.

\section{Limberger}

Variété de vigueur moyenne et de bonne fertilité. Débourrement précoce. Originaire d'Autriche, s'est répandue dans le Würtemberg et a été introduite en Slovaquie (synonymes : Blaufränkish et Frankovka). En France, a été rencontrée en Auvergne, dans l'Ain, l'Yonne et la région de Toul. 
TABLEAU 3

Résultats du top-cross (Année 1977)

Top-cross results

\begin{tabular}{|c|c|c|c|c|c|}
\hline Parent femelle & Parent mâle & $\begin{array}{c}\mathrm{Nb} \text { de } \\
\text { grappes } \\
\text { pollinisées }\end{array}$ & $\begin{array}{l}\mathrm{Nb} \text { de } \\
\text { pépins } \\
\text { obtenus }\end{array}$ & $\begin{array}{c}\text { p. } 100 \\
\text { germination }\end{array}$ & $\begin{array}{c}\text { Nb de } \\
\text { plantes } \\
\text { disponibles }\end{array}$ \\
\hline \multirow[t]{16}{*}{ Cabernet-Sauvignon } & $\times$ Carmenere & 10 & 623 & 59 & 245 \\
\hline & $\times$ Saint-Laurent & 6 & 560 & 63 & 315 \\
\hline & $\times$ Colorino & 5 & 600 & 57 & 311 \\
\hline & $\times$ Saperavi & 5 & 439 & 50 & 206 \\
\hline & $\times$ Manseng noir & 4 & 600 & 52 & 288 \\
\hline & $\times$ Limberger & 4 & 600 & 66 & 340 \\
\hline & $\times$ Fogarina & 6 & 360 & 73 & 247 \\
\hline & $\times$ Soinouri & 5 & 582 & 67 & 360 \\
\hline & $\times$ Touriga & 7 & 590 & 79 & 424 \\
\hline & $\times$ Argant & 6 & 380 & 65 & 230 \\
\hline & $\times$ Calabrese & 5 & 350 & 80 & 277 \\
\hline & $\times$ Refoscone & 6 & 381 & 70 & 288 \\
\hline & $\times$ Souzao & 4 & 608 & 40 & 191 \\
\hline & $\times$ Abouriou & 4 & 600 & 47 & 289 \\
\hline & $\times$ Joubertin & 5 & 252 & 62 & 120 \\
\hline & $\times$ Terrano & 5 & 525 & 69 & 310 \\
\hline \multirow[t]{16}{*}{ Grenache noir } & $\times$ Carmenere & 6 & 319 & 80 & 234 \\
\hline & $\times$ Saint-Laurent & 7 & 213 & 82 & 163 \\
\hline & $\times$ Colorino & 5 & 373 & 78 & 257 \\
\hline & $\times$ Saperavi & 6 & 396 & 73 & 259 \\
\hline & $\times$ Manseng noir & 4 & 389 & 74 & 270 \\
\hline & $\times$ Limberger & 4 & 492 & 83 & 363 \\
\hline & $\times$ Fogarina & 5 & 382 & 68 & 227 \\
\hline & $\times$ Soinouri & 7 & 434 & 76 & 266 \\
\hline & $\times$ Touriga & 4 & 375 & 84 & 265 \\
\hline & $\times$ Argant & 6 & 397 & 73 & 276 \\
\hline & $\times$ Calabrese & 4 & 530 & 81 & 394 \\
\hline & $\times$ Refoscone & 7 & 231 & 72 & 159 \\
\hline & $\times$ Souzao & 5 & 428 & 77 & 297 \\
\hline & $\times$ Abouriou & 6 & 363 & 71 & 257 \\
\hline & $\times$ Joubertin & 3 & 503 & 83 & 421 \\
\hline & $\times$ Terrano & 7 & 257 & 83 & 199 \\
\hline
\end{tabular}

\section{Manseng noir}

Variété vigoureuse et fertile, peu sensible à la pourriture. Aire de culture limitée aux coteaux entre les Graves de Pau et d'Oloron (Pyrénées atlantiques) où ce cépage est souvent dominant. Donne un vin riche en tannins.

\section{Refoscone}

Variété de vigueur et de fertilité moyennes, à grappes lâches, peu sensibles à la pourriture. Débourrement précoce et maturité assez tardive. Cultivée autrefois dans le Frioul, mais aujourd'hui presque disparue.

\section{Saint-Laurent}

Variété très précoce, assez fertile, qui n'est plus cultivée actuellement. A été cultivée dans le Würtemberg et le Palatinat, introduite en Moravie et Slovaquie ; en France, a été rencontrée dans la région de Toul. Dans les vignobles septentrionaux, donnerait un meilleur vin que le Portugais bleu.

\section{Saperavi}

Variété peu vigoureuse, de fertilité moyenne, à grappes petites et assez lâches, peu sensibles à la pourriture. Faible productivité. Débourrement précoce. Culture limitée à son aire d'origine : la Kakhétie.

\section{Soinouri}

Variété de vigueur et fertilité moyennes, à grappes petites et peu sensibles à la pourriture grise. Faible productiviţé et maturité tardive. Cultivée en Georgie occidentale pour la production de vins de table.

\section{Souzao}

Variété de vigueur moyenne et de fertilité assez bonne. Débourrement tardif. Vinhao, Negrao et Souzon sont des synonymes de ce cépage qui est surtout cultivé dans le Douro et au nord du Portugal jusque dans la région des vins verts.

\section{Terrano}

Variété vigoureuse mais à fertilité assez faible, à grappes petites peu sensibles à la pourriture. Maturité tardive. Débourrement moyen comme le Grenache. Cultivée dans les zones karstiques de Gorizia et de Trieste, elle se retrouve dans les provinces de Forli et de Ravenne, en Emilie.

\section{Touriga}

Variété de vigueur moyenne, pas très fertile, à grappes plutôt petites. Aire de culture étendue au Portugal : Traz os Montes, Douro et Dao en particulier où elle prédomine parmi les cépages rouges. 


\section{REFERENCES BIBLIOGRAPHIQUES}

Bouquet A., 1977. Amélioration génétique de la Vigne : essai de définition d'un schéma de sélection applicable à la création de nouvelles variétés. Ann. Amélior. Plantes, 27 (1), 75-86.

Bouquet A., 1980. Vitis $\times$ Muscadinia hybridization : a new way in grape breeding for disease resistance in France. Comm. IIIrd Intern. Symp. on grape breeding. Davis (Calif.), June 1980.

Carbonneau A., Pouget R., Chaves M. M. et Troussard J. L., 1980. Early physiological selection tests based on leaf activity. Comm. IIIrd Intern. Symp. on grape breeding. Davis (Calif.), June 1980. Demarly, Y., 1972. Commentaires sur les aptitudes à la combinaison. Ann. Amélior. Plantes, 22 (2), 187-200.

Doazan J. P., Kim S. K., 1978. Recherche de génotypes résistants au Mildiou dans des croisements interspécifiques. In : Génétique et Amélioration de la Vigne. I.N.R.A. Ed., 243-249.

Durquety P. M., 1978. Les progrès réalisés dans l'Ouest de la France par l'amélioration génétique de Vitis vinifera L. In : Génétique et Amélioration de la Vigne, I.N.R.A. Ed., 369-376.

Gallais A., 1977. Amélioration des populations, méthodes de sélection et création de variétés. I. Synthèse sur les problèmes généraux et sur les bases théoriques pour la sélection récurrente intrapopulation. Ann. Amélior. Plantes, 27 (3) 281-329.

Gordon G. H., 1980. A method of parental selection and cross prediction using incomplete partial diallels. Part 1: a simulation study. T.A.G., 56, 225-232.
Hewitt W. B., Rives M., 1979. On the preservation and use of the genetic resources in the genus Vitis. Ann. Amélior. Plantes, 29 (5) 515-522

Pandey K. K., 1972. Origin of genetic variation : regulation of genetic recombination in the higher organisms. A theory. T.A.G., 42, 250-261.

Pederson D. G., 1980. The augmented partial diallel cross. Heredity, 44, 327-331.

Pouget R., Ottenwaelter M., 1978. Etude de l'adaptation de nouvelles variétés de porte-greffes à des sols très chlorosants. Comm. Symp. Intern. sur l'Ecologie de la Vigne. Constanta (Roumanie).

Rives M., 1977. Réflexions pour une stratégie de l'amélioration de la Vigne. Ann. Amélior. Plantes, 27 (1), 61-73.

Srinivasan C., Mullins M. G., 1979. Flowering in Vitis : conversion of tendrils into inflorescences and bunches of grapes. Planta, 145, 187-192.

Truel P., Vergnes A., 1978. Obtention de variétés de cuve et de table adaptées aux conditions du vignoble méridional. In : Génétique et Amélioration de la Vigne, I.N.R.A. Ed., 361-368.

Wagner R., Bronner A., 1974. Etude de la fertilité des semis de Vitis vinifera $\mathrm{L}$. : Application à la mise au point d'un test précoce de sélection. Ann. Amélior. Plantes, 24 (2), 145-157. 